\title{
SOME RESULTS ON NONNEGATIVELY CURVED MANIFOLDS
}

\author{
WALTER A. POOR, JR.
}

Cheeger and Gromoll have proven [1] that every complete Riemannian manifold $M$ of nonnegative sectional curvature has a compact totally convex totally geodesic submanifold $S$, which they called a soul of $M$. Furthermore, they have proven that $M$ is homeomorphic to the normal bundle $\nu(S)$ of $S$ in the tangent bundle $T M$ of $M$; for $M$ simply connected and of dimension greater than five, using the open $h$-cobordism theorem they proved that $M$ and $\nu(S)$ are diffeomorphic. This result greatly simplifies the study of noncompact complete manifolds of nonnegative curvature since it partially reduces the problem to the study of vector bundles over compact manifolds.

Our first result will be to prove the differentiable soul theorem, that is, we will prove that $M$ and $\nu(S)$ are diffeomorphic (without dimension or connectivity assumptions). The diffeomorphism to be constructed will be rather closely adapted to the geometric situation.

In preparation for the statement of the second result, we recall that if $\chi$ is the Euler form on an oriented complete even-dimensional Riemannian manifold $M$, then $\int_{M} \chi$ (if it exists) is called the total curvature of $M$. It has long been known [4] that the total curvature of a complete surface of nonnegative curvature is less than or equal to one; since in this case $K=2 \pi \chi$, where $K$ is the sectional curvature, the result is usually written

$$
\int_{M} K \leq 2 \pi
$$

Our second result will be to prove that for an oriented complete nonnegatively curved Riemannian manifold $M$ of dimension 4, the total curvature exists and is bounded between zero and the Euler characteristic of $M$. This obviously generalizes both the above result for surfaces and the generalized Gauss-Bonnet theorem for four-dimensional oriented compact manifolds of nonnegative curvature. In addition, for oriented complete nonnegatively curved Riemannian manifolds of even dimension greater than 4, the argument reduces the question to the so-called algebraic Hopf conjecture. This implies a partial result in dimension 6 .

Received July 12, 1973. 
The results are related in the sense that both rest heavily on the global existence of certain convex functions; the analysis of these functions, which are in general only continuous, is the central technical aspect of the work.

Finally we give some applications.

This paper presents the essence of the author's doctoral dissertation at the State University of New York at Stony Brook under the direction of Detlef Gromoll. Here the author would like to express his thanks to Professor Gromoll and also to the State University of New York at Stony Brook for its support.

\section{Preliminaries}

Given a $C^{\infty}$ manifold $M=M^{n}, T M \stackrel{\pi}{\longrightarrow} M$ will denote the tangent bundle. For $p$ in $M, M_{p}:=\pi^{-1}(p)$ is the tangent space to $M$ at $p . \Gamma \xi$ will be the set of $C^{\infty}$ sections in the bundle $\xi \rightarrow M$; particular examples are $F M:=\Gamma(M \times R)$ $=\left\{C^{\infty}\right.$ functions on $\left.M\right\}$, and $\mathfrak{b} M:=\Gamma T M=\left\{C^{\infty}\right.$ vector fields on $\left.M\right\} . \nabla$ will always denote the covariant derivative operator $\mathfrak{v} M \times \Gamma \xi \rightarrow \Gamma \xi$ in whatever bundle $\xi$ we are discussing; exp will be the exponential map of $\nabla$.

If $M$ is a Riemannian manifold, then $\langle$,$\rangle will be the first fundamental form,$ and $\nabla: \mathfrak{v} M \times \mathfrak{b} M \rightarrow \mathfrak{b} M$ will denote the Levi-Civita connection. $T_{1} M:=$ $\{u \in T M \mid\|u\|=1\}$ is the (unit) tangent sphere bundle, and for $p$ in $M, S_{p}^{n-1}$ $:=M_{p} \cap T_{1} M$ is the (unit) tangent sphere to $M$ at $p$. Given nonzero $u$ and $v$ in $M_{p}, \Varangle(u, v)$ will denote the angle in $[0, \pi]$ between $u$ and $v$.

For $p$ and $q$ in $M, \rho(p, q)$ is the metric distance from $p$ to $q$. For $r>0$ and $p$ in $M, B_{r}(p)$ is the open metric ball $\{q \in M \mid \rho(p, q)<r\}$ with boundary equal to the metric sphere $S_{r}(p):=\{q \in M \mid \rho(p, q)=r\}$. A geodesic is said to be normal if it is parametrized by arc length. If there exists a unique normal geodesic $c:[0, \rho(p, q)] \rightarrow M$ from $p$ to $q$, it will be called the minimal connection from $p$ to $q$.

Let $\chi_{M}$ be the Euler characteristic of $M$.

In addition to the construction of a soul of $M$, we will also need a few of the technical facts which Cheeger and Gromoll used in the proof of the continuous soul theorem; this section consists mainly of the necessary material from [1], which we reformulate for our purposes.

A nonempty subset $C$ of $M$ will be said to be totally convex if for any $p$ and $q$ in $C$ and any geodesic $c:[0,1] \rightarrow M$ from $p$ to $q, c$ lies in $C$. $C$ is strongly convex if for any $p$ and $q$ in $C$, there is a minimal connection $c$ from $p$ to $q$ and $c$ lies in $C$.

Lemma 1. Let $C$ be a closed totally convex subset of an arbitrary Riemannian manifold $M$. Then $C$ has the structure of an imbedded k-dimensional submanifold of $M$ with smooth totally geodesic interior $N$ and (possibly nonsmooth) boundary $\partial C=\bar{N}-N$.

Cheeger and Gromoll actually proved a stronger version of the lemma, but this is all we need. 
For a closed totally convex set $C$ and for $a \geq 0$, let ${ }^{a} C:=\{p \in M \mid \rho(p, C)$ $\leq a\}=\bigcup_{p \in C} B_{a}(p)$; in addition, if $\partial C \neq \emptyset$, let $C^{a}:=\{p \in C \mid \rho(p, \partial C) \geq a\}$. Notice that $C^{a}$ may be empty.

Lemma 2. Let $M$ have nonnegative curvature, and let $C$ be a closed totally convex subset of $M$ with nonempty boundary. If $C^{a}$ is nonempty, then $C^{a}$ is a closed totally convex set. If $a_{\max }:=\max \left\{a \in R \mid C^{a} \neq \emptyset\right\}=\max _{p \in C} \rho(p, \partial C)$, then $\operatorname{dim} C^{a_{\max }}<\operatorname{dim} C$.

Lemma 3 (standard). For every compact subset $K$ of $M$ there is a number $\varepsilon>0$ such that for all $p$ in $K$ and $r$ in $(0, \varepsilon)$ :

(1) the metric ball $B_{r}(p)$ is the diffeomorphic strongly convex image under $\exp _{p}$ of the open Euclidean ball of radius $r$ about 0 in $M_{p}$;

(2) if $c:[0, u] \rightarrow B_{r}(p)$ is a nonconstant geodesic, $c_{0}:[0,1] \rightarrow B_{r}(p)$ is the minimal geodesic from $p$ to $c(0)$, and $\left\langle\dot{c}(0), \dot{c}_{0}(1)\right\rangle \geq 0$, then the function $s \mapsto \rho(c(s), p)$ is strictly increasing on $[0, u]$.

From now on, we will always assume that $M$ is a complete noncompact Riemannian manifold of nonnegative curvature.

We will only use parts (1) and (2) of the next lemma; part (3) is presented because it gives the flavor of the proof of the continuous soul theorem and is a model for some of the work we must do.

Lemma 4. Let $K$ be a compact totally convex set in $M$ with nonempty boundary, and let $0<\varepsilon \leq a_{\max }$, where $\varepsilon$ is a constant as in Lemma 3, and $a_{\max }=\max \{\rho(p, \partial K) \mid p \in K\}$. Then

(1) there exists $\delta \in(0, \varepsilon)$ such that $\rho\left(p, K^{a^{\prime}}\right)<\varepsilon$ for all $p$ in $K^{a}$ if $0 \leq a \leq$ $a^{\prime} \leq a_{\max }$ and $a^{\prime}-a<\delta$

(2) there exists a "continuous geodesic contraction" of $K^{a}$ onto $K^{a^{\prime}}$ if $0 \leq a \leq a^{\prime} \leq a_{\max }$ and $a^{\prime}-a<\delta$;

(3) given $0 \leq a<a^{\prime}<a_{\max }$ such that $a^{\prime}-a<\delta$, there is a homeomorphism of $\partial K^{a} \times[0,1]$ onto $K^{a}-$ int $K^{a^{\prime}}$.

We will now describe the constructions in parts (2) and (3) explicitly.

Given $p$ in $K^{a}-K^{a^{\prime}}$, there is a closest point $q$ in $K^{a^{\prime}} ; q$ is unique by the first variation formula and Lemma 3. Define $h(p):=q . h$ is continuous by uniqueness of $q$ and the continuity of $p \mapsto \rho\left(p, K^{a^{\prime}}\right)$. Thus we can contract $K^{a}$ onto $K^{a^{\prime}}$ along the minimal connections from points on $\partial K^{a}$ to the closest points on $K^{a^{\prime}}$. We will occasionally refer to $h$ as "the contraction" onto $K^{a^{\prime}}$.

Let $a^{\prime}<a^{\prime \prime}<a_{\max }$ so that $a^{\prime \prime}-a<\delta$. Let $\tilde{h}$ be the contraction onto $K^{a^{\prime \prime}}$, and for $p$ in $\partial K^{a}$ let $g_{p}:[0,1] \rightarrow K^{a}$ be the unique minimal geodesic such that $g_{p}(0)=p$ and $g_{p}(1)=\tilde{h}(p)$. Define $\Phi: \partial K^{a} \rightarrow(0,1)$ such that $g_{p}(\Phi(p)) \in \partial K^{a^{\prime}} ;$ $\Phi$ is continuous. Define $F: \partial K^{a} \times[0,1] \rightarrow K^{a}-$ int $K^{a^{\prime}}$ such that $(p, t) \mapsto$ $g_{p}(\Phi(p) t) . F$ is the homeomorphism.

Let $\left\{C_{t}\right\}_{t \geq 0}$ be a family of nonempty compact totally convex sets such that

(1) $\operatorname{dim} C_{t} \begin{cases}<\operatorname{dim} M & \text { if } t=0 \\ =\operatorname{dim} M & \text { if } t>0\end{cases}$ 
(2) $t_{2} \geq t_{1} \Rightarrow C_{t_{1}}=C_{t_{2}}^{t_{2}-t_{1}}$

(3) $\cup_{t \geq 0} C_{t}=M$.

Note that for all $t>0, C_{t}{ }^{t}=C_{0}$.

Cheeger and Gromoll constructed this family as follows: a normal geodesic $c:[0, \infty) \rightarrow M$ is called a ray if each segment of $c$ is minimal between its endpoints. It is straightforward to see that every point in any noncompact complete Riemannian manifold has at least one ray through it. If $c$ is a ray, let $B_{c}:=\bigcup_{t>0} B_{t}(c(t)) . B_{c}$ is called the open half-space with respect to $c$, and its complement $M-B_{c}$ is totally convex in our case.

Now pick $p$ in $M$. For $t \geq 0$, let $L_{t}:=\bigcap_{c}\left(M-B_{c_{t}}\right)$, where the intersection is taken over all rays $c$ emanating from $p$ and $c_{t}$ is the ray determined by $c_{t}(s):=c(t+s), 0 \leq s<\infty$. Each $L_{t}$ is compact and totally convex, and $p$ is in $\partial L_{0}$.

If $\operatorname{dim} L_{0}<\operatorname{dim} M$, then let $C_{t}=L_{t}$ for all $t \geq 0$. If $\operatorname{dim} L_{0}=\operatorname{dim} M$, let $a_{0}:=\max _{q \in L_{0}} \rho\left(q, \partial L_{0}\right)$ and

$$
C_{t}= \begin{cases}L_{0}^{a_{0}-t} & \text { for } 0 \leq t \leq a_{0} \\ L_{t-a_{0}} & \text { for } a_{0}<t\end{cases}
$$

It $\partial C_{0}=\emptyset$, then let $S=C_{0}$. If $\partial C_{0} \neq \emptyset$, let $K_{0}:=C_{0}$ and $a_{0}:=$ $\max _{p \in K_{0}} \rho\left(p, \partial K_{0}\right)$; thus $K_{1}:=K_{0}^{a_{0}}$ is a totally convex set of lower dimension than $K_{0}$. Iteration yields a sequence $K_{0} \supset K_{1} \supset \cdots \supset K_{J}$ of compact totally convex sets such that $K_{i+1}:=K_{i}^{a_{i}}$, where $a_{i}:=\max \left\{\rho\left(p, \partial K_{i}\right) \mid p \in K_{i}\right\}$, and such that $\partial K_{J}=\emptyset ; J<\operatorname{dim} M$. Let $S:=K_{J}$.

Cheeger and Gromoll called $S$ a soul of $M$; it is unique up to the choice of $p$. They proved that $M$ is homeomorphic to the normal bundle of $S$, and stated that the construction could be smoothed out so as to give a diffeomorphism. We have carried out this process in detail, and consider this approach to be unnecessarily complicated. Here we present a modified construction which is simpler and also more satisfying geometrically.

\section{The differentiable soul theorem}

Let $M$ be a complete noncompact Riemannian manifold of nonnegative sectional curvature.

Theorem A. $M$ is diffeomorphic to $\nu(S)$.

The exponential map restricted to $\nu(S)$ is a smooth surjective map onto $M$, which fails to be injective. The map we obtain is modelled (roughly speaking) on the exponential map, and in fact we may think of it as being a smooth bijective "bent exponential map".

We will actually prove the following theorem; by standard arguments from differential topology it is equivalent to Theorem A. For these arguments, as 
well as for several arguments used in the proof of the following theorem, we refer the reader to references [8] and [10].

Theorem B. Let $M_{0}=S$. Then there exists an increasing sequence $M_{1}, M_{2}$, .. of nonempty compact submanifolds with boundary such that

(1) $M_{1}$ is diffeomorphic to a disc bundle in $\nu(S)$,

(2) $M_{i+1}-$ int $M_{i}$ is diffeomorphic to $\partial M_{i} \times[0,1]$ for $i \geq 1$, and

(3) $M$ is the (disjoint) union $S \cup\left(\cup_{i \geq 0}\left(M_{i+1}-M_{i}\right)\right)$.

This result will be proven in several steps. First we make a few remarks.

Recall that we have an expanding sequence $\left\{C_{t}\right\}_{t \geq 0}$ of nonempty totally convex compact sets and a decreasing sequence $C_{0}=K_{0} \supset K_{1} \supset \cdots \supset K_{J}=S$ as constructed in $\S 1$.

Cheeger and Gromoll construct a continuous "broken exponential map" by locally adjusting the exponential map restricted to $\nu(S)$ to get a locally injective map; by using the above sequences of sets to keep track of the resulting "continuous flow", they end up with a homeomorphism from $\nu(S)$ to $M$.

The local arguments are of three types. First, given a sufficiently small $r>0$ they construct a homeomorphism between ${ }^{r} K_{0}$ and the disc bundle $\nu_{r}(S)$ of radius $r$. This argument takes into account the difference in dimension between $K_{i}$ and $K_{i+1}$ at each step. Then they use another argument to show that ${ }^{r} C_{0}$ is homeomorphic to $C_{r}=C_{1}{ }^{1-r}$. The third argument uses the exhaustive sequence $\left\{C_{t}\right\}$ to extend this homeomorphism globally.

Simply smoothing out these constructions directly is not very efficient, and so we have modified the whole approach.

First we present an interesting fact.

Lemma 5. Let $K$ be a compact totally convex subset of $M$. Let $d: M \rightarrow R$ such that $d(p):=\rho(p, K)$. Then there exists an open neighborhood $U$ of $K$ such that $\left.d\right|_{U-K}$ is $C^{1}$.

Proof. Consider the $\operatorname{set}^{1} K$. Choose $\varepsilon$ in $(0,1]$ for ${ }^{1} K$ as in Lemma 3. Then $\varepsilon$ also satisfies the conclusion of Lemma 3 for the smaller set ${ }^{\circledR} K$. Let $U:=$ int ${ }^{\circ} K$. Let $h: U \rightarrow K$ be the "continuous geodesic contraction" onto $K$ given in Lemma $4(3) ; h(p)$ is the unique point in $K$ such that $\rho(p, K)=\rho(p, h(p))$ $=d(p)$.

Let $p \in A:=U-K$. Define $f_{p}: A \rightarrow R$ such that $f_{p}(q):=\rho(q, h(p))$. Clearly we have $f_{p} \geq d$; in addition, $f_{p}(p)=d(p)$, and $f_{p}$ is $C^{\infty}$ on a neighborhood of $p$ in $A$. Let $\Delta \subset M_{h(p)}$ be the supporting hyperplane for $K$ orthogonal to the minimal connection from $p$ to $h(p)$ (cf. [1]). Let $\Delta_{\varepsilon}:=\{v \in \Delta \mid\|v\|$ $<\varepsilon\}$. Then $\exp _{h(p)} \Delta_{\varepsilon}$ is a local supporting hypersurface for $K$. For $q \in A$, let $g_{p}(q):=\rho\left(q, \exp _{h(p)} \Delta_{\varepsilon}\right)$. Then $g_{p} \leq d, g_{p}(p)=d(p)$, and $g_{p}$ is $C^{\infty}$ on a neighborhood of $p$ in $A$.

By the squeezing principle, $d$ is (once) differentiable at $p$, and in fact

$$
d_{* \mid p}=f_{p^{*} \mid p}=g_{p^{*} \mid p}
$$


To see this, let $c$ be a $C^{\infty}$ curve in $A$ such that $c(0)=p$. For small positive $t$,

$$
\frac{1}{t}\left[g_{p} \circ c(t)-g_{p}(p)\right] \leq \frac{1}{t}[d \circ c(t)-d(p)] \leq \frac{1}{t}\left[f_{p} \circ c(t)-f_{p}(p)\right],
$$

and therefore,

$$
\left(g_{p} \circ c\right)^{\prime}(0) \leq \lim _{t \rightarrow 0+} \frac{1}{t}[d \circ c(t)-d(p)] \leq\left(f_{p} \circ c\right)^{\prime}(0) .
$$

Similarly,

$$
\left(f_{p} \circ c\right)^{\prime}(0) \leq \lim _{t \rightarrow 0^{-}} \frac{1}{t}[d \circ c(t)-d(p)] \leq\left(g_{p} \circ c\right)^{\prime}(0) .
$$

Thus $\left(f_{p} \circ c\right)^{\prime}(0)=\left(g_{p} \circ c\right)^{\prime}(0)$, and therefore $(d \circ c)^{\prime}(0)$ exists and equals $\left(f_{p} \circ c\right)^{\prime}(0)$.

Since the assignment $p \mapsto h(p)$ is continuous, so is $p \mapsto \nabla f_{p}(p)=\nabla d(p)$ (see the remark below); thus $d$ is $C^{1}$ on $U-K$.

Remark. Given $p \in U-K$, let $\gamma_{p}$ be the minimal connection from $p$ to $h(p) \in K$. Then

$$
\nabla d \circ \gamma_{p}(t)=\nabla f_{p} \circ \gamma_{p}(t)=-\dot{\gamma}_{p}(t)
$$

for $0 \leq t<d(p)$. In particular, $\nabla d$ is a continuous autoparallel unit vector field on $U-K$. In addition, $h(p)=\exp _{p}(-d(p) \nabla d(p))$.

Lemma 6. Let $K$ be a compact totally convex subset of $M$ with nonempty boundary, and let $a_{\max }:=\max \{\rho(p, \partial K) \mid p \in K\}$. Choose $\varepsilon \in(0,1]$ as in the proof of Lemma 5 , and choose $r \in(0, \varepsilon)$. Define $d:\left[0, a_{\max }\right] \times{ }^{r} K \rightarrow R$ such that $d(a, p)=d_{a}(p):=\rho\left(p, K^{a}\right)$, and set $(\nabla d)(a, p)=\nabla d_{a}(p)$. Then $\nabla d$ is uniformly continuous on the set

$$
A:=\left\{(a, p) \in\left[0, a_{\max }\right] \times{ }^{r} K \mid p \in{ }^{r}\left[K^{a}\right]-\text { int }^{r / 4}\left[K^{a}\right]\right\} .
$$

Proof. First we prove that $d$ is continuous on $\left[0, a_{\max }\right] \times{ }^{r} K$; we already know that for each $a, d_{a}$ is continuous on ${ }^{r} K$ and $C^{1}$ on ${ }^{r}\left[K^{a}\right]-K^{a}$.

Let $\left\{\left(a_{i}, p_{i}\right)\right\}$ be a sequence in $\left[0, a_{\max }\right] \times{ }^{r} K$ converging to $(a, p)$. Choose a sequence $\left\{q_{i} \in K^{a_{i}}\right\}$ such that $d\left(a_{i}, p_{i}\right)=\rho\left(p_{i}, q_{i}\right)$. Let $q$ be a limit point of this sequence; such a $q$ exists since $K$ is compact. Then $\lim _{i} d\left(a_{i}, p_{i}\right)=\lim _{i} \rho\left(p_{i}, q_{i}\right)$ $=\rho(p, q) \geq d(a, p)$. Now choose $\bar{q} \in K^{a}$ such that $\rho(p, \bar{q})=d(a, p)$, and let $\left\{\bar{q}_{i}\right\}$ be a sequence such that $\bar{q}_{i} \in K^{a_{i}}$ and $\lim _{i} \bar{q}_{i}=\bar{q}$. For each $i, d\left(a_{i}, p_{i}\right) \leq$ $\rho\left(p_{i}, \bar{q}_{i}\right)$, and therefore $\lim _{i} d\left(a_{i}, p_{i}\right) \leq \lim _{i} \rho\left(p_{i}, \bar{q}_{i}\right)=\rho(p, \bar{q})=d(a, p)$. Thus $d$ is continuous.

But then since $A=d^{-1}\left[\frac{1}{4} r, r\right], A$ must be compact. Define $h: A \rightarrow K$ such 
that $h(a, p)$ is the unique point $q$ in $K^{a}$ for which $\rho(p, q)=d(a, p)$. By continuity of $d, h$ is also continuous. But

$$
h(a, p)=\exp _{p}(-d(a, p) \nabla d(a, p)),
$$

and thus $\nabla d$ is continuous on the set $A$.

We are now ready to prove Theorem $B$.

Choose $\varepsilon_{1} \in(0,1)$ for the set $C_{1}$ as in Lemma 3. Choose $\tilde{\delta}_{1} \in(0, \varepsilon)$ as in Lemma 4. Let $0<r<\tilde{\delta}_{1}$, and choose $\delta_{1}$ for $\varepsilon=\frac{1}{4} r$ and the set $C_{1}$ as in Lemma 4. Recall that $K_{0}=C_{0}$ and $K_{i+1}=K_{i}{ }^{a_{i}}$, where $a_{i}=\max \left\{\rho\left(p, \partial K_{i}\right) \mid p \in K_{i}\right\}$.

Since $\nabla d$ is uniformly continuous on the set

$$
A:=\left\{(a, p) \in\left[0, a_{0}\right] \times{ }^{r} K_{0} \mid p \in{ }^{r}\left[K_{0}{ }^{a}\right]-\text { int }^{r / 4}\left[K_{0}^{a}\right]\right\},
$$

there exists $\xi>0$ such that if $(a, p)$ and $(b, p) \in A$ and $|a-b|<\xi$, then $\Varangle\left(\nabla d_{a}(p), \nabla d_{b}(p)\right)<\frac{1}{6} \pi$; we may assume that $\xi<\delta_{1}$.

Choose numbers $0=b_{0}<b_{1}<\cdots<b_{k}=a_{0}$ such that $b_{i+1}-b_{i}<\xi$, $0 \leq i \leq k-1$. Let $L_{0}=K_{0}, L_{1}=K_{0}^{b_{1}}, \cdots, L_{k}=K_{0}^{b_{k}}=K_{1}$. Continue similarly with $K_{1}, \cdots, K_{J-1}$ (choosing a different value of $\xi$ at each step, if necessary) to get a decreasing sequence

$$
K_{0}=L_{0} \supset L_{1} \supset \cdots \supset L_{k}=K_{1} \supset \cdots \supset K_{2} \supset \cdots \supset L_{\alpha}=S
$$

such that for $1 \leq i \leq \alpha, L_{i}=L_{i-1}^{a_{i}}$ for some sufficiently small $a_{i}>0$, and

$$
{ }^{r / 4} L_{i-1} \subset{ }^{r / 2} L_{i} \subset{ }^{r / 2} L_{i-1} .
$$

Let $d_{i}:{ }^{r} L_{i} \rightarrow R$ be the distance function from ${ }^{r} L_{i}$ to $L_{i}$. Then each $d_{i}$ is $C^{1}$ off of $L_{i}$, and if $p \in{ }^{r} L_{i-1}-{ }^{r / 2} L_{i}$, then $\Varangle\left(\nabla d_{i}(p), \nabla d_{i-1}(p)\right)<\frac{1}{6} \pi$.

We can choose a $C^{\infty}$ function $\tilde{d}_{i}$ on ${ }^{r} L_{i}$ such that

(1) $\left|d_{i}-\tilde{d}_{i}\right|<\frac{1}{8} r / \alpha$,

(2) $\nabla \tilde{d}_{i} \neq 0$ and $\Varangle\left(\nabla d_{i}, \nabla \tilde{d}_{i}\right)<\frac{1}{6} \pi$ off of $r / 8 L_{i}$.

For $1 \leq i \leq \alpha$, let $N_{i}$ be the compact submanifold $\left\{p \in{ }^{r} L_{i} \mid \tilde{d}_{i}(p) \leq\right.$ $\left.\frac{4 \alpha-2 i+1}{4 \alpha} r\right\}$ with $C^{\infty}$ boundary $H_{i}=\tilde{d}_{i}^{-1}\left(\frac{4 \alpha-2 i+1}{4 \alpha} r\right)$. Then

$$
{ }^{\sigma} L_{i} \subset \text { int } N_{i} \subset N_{i} \subset \text { int }{ }^{\tau} L_{i},
$$

where $\sigma=\left(\frac{1}{2}(2 \alpha-i) / \alpha\right) r$ and $\left.\tau=\left(\frac{1}{2}(2 \alpha-i+1) / \alpha\right) r\right)$. Also, $\nabla \tilde{d}_{i} \perp H_{i}$. Thus $H_{i}$ is also transverse to $\nabla \tilde{d}_{i-1}, 1 \leq i \leq \alpha$, by the way we chose everything. Therefore the gradient flow of $\tilde{d}_{i-1}$ determines (after reparametrization) a diffeomorphism $H_{i} \times[0,1] \cong N_{i-1}-$ int $N_{i}, 2 \leq i \leq \alpha$.

In addition, $\nabla \tilde{\delta}_{\alpha}$ determines a diffeomorphism between $\partial\left({ }^{r / 2} S\right) \times[0,1]$ and 
$N_{\alpha}-$ int ${ }^{r / 2} S$. By the choice of $r$, the exponential map is a diffeomorphism between the normal disc bundle $\nu_{r / 2}(S)$ of radius $\frac{1}{2} r$ and ${ }^{r / 2} S$.

Set

$$
M_{1}:={ }^{r / 2} S, \quad M_{2}:=N_{\alpha}, \cdots, \quad M_{\alpha+1}:=N_{1} .
$$

$M_{\alpha+1}$ is a compact submanifold with boundary such that

$$
{ }^{r_{1}} L_{1} \subset M_{\alpha+1} \subset{ }^{r} L_{1}
$$

where $r_{1}=\left(\frac{1}{2}(2 \alpha-1) / \alpha\right) r$. We will now show how to find the larger submanifolds required by the theorem.

Choose $\varepsilon_{2} \in(0,1)$ for the set $C_{2}$ as in Lemma $3, \tilde{\delta}_{2} \in\left(0, \varepsilon_{2}\right)$ for $C_{2}$ as in Lemma 4 , and let $0<r_{2}<\min \left\{\tilde{\delta}_{2}, r_{1}\right\}$; finally, choose $\delta_{2}$ for $\varepsilon=r_{2}$ as in Lemma 4 for the set $C_{2}$.

Let $\kappa$ be an integer greater than $1 / \delta_{2}$. Then $\left(r_{1}-r_{2}\right) / \kappa<1 / \kappa<\delta_{2}$. For $j=0,1, \cdots, \kappa$, let $\eta(j):=r_{1}-j\left(r_{1}-r_{2}\right) / \kappa$. As $j$ runs from 0 to $\kappa, \eta(j)$ decreases from $r_{1}$ to $r_{2}$; let $\ell(j)=j / \kappa$. We have the following inclusions:

$$
C_{\ell(j-1)} \subset C_{\ell(j)} \subset{ }^{\eta(j)} C_{\ell(j-1)} \subset{ }^{\eta(j-1)} C_{\ell(j-1)} \subset{ }^{\eta(j)} C_{\ell(j)}
$$

By the way we have chosen everything, we can now use the appropriate distance functions as before to obtain compact submanifolds $M_{i}$ with boundary for $i=\alpha+2, \cdots, \alpha+\kappa-1$, such that

$$
{ }^{\eta(j)} C_{\ell(j)} \subset M_{\alpha+j+2} \subset{ }^{\eta(j+1)} C_{\ell(j+1)}
$$

for $j=0, \cdots, \kappa-1$, and having the properties required in the theorem.

$M_{\alpha+\kappa-1}$ is now close to ${ }^{r_{2}} C_{1} \subset C_{2}$. Pick all the appropriate constants for $C_{3}$ and iterate. This completes the argument.

\section{Total curvature of $M$}

In this section we will prove that for a complete noncompact oriented Riemannian manifold $M$ of dimension 4, the total curvature of $M$ exists and is bounded between 0 and the Euler characteristic of $M \cdot{ }^{1}$ In addition, if the dimension of $M$ is $2 k, k \geq 3$, then our argument reduces the problem to the algebraic Hopf conjecture; in particular, this implies a partial result in dimension 6.

The calculus we employ is not new, but does not appear in the literature in this exact form. We start by presenting classical material to help fix the notation.

Let $\pi^{*} T M$ be the pullback of $T M$ over itself under the map $\pi$ :

\footnotetext{
${ }^{1}$ This result has also been proved by $\mathbf{R}$. Walter using a different approach.
} 


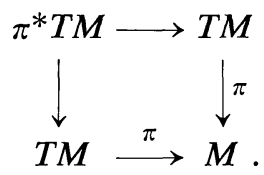

We may realize $\pi^{*} T M$ as the vertical bundle $V M$ in $T T M$ : for $v \in T M$, the fiber in $V M$ over $v$ is $\left.\pi_{*}\right|_{v} ^{-1}(0) \subset T T M$. For $u$ in $M_{p}$, let $u_{\#}$ denote the canonical identification of $M_{p}$ with $\left(M_{p}\right)_{u} \subset V M$ by parallel transport, that is, for $v \in M_{p}, u_{\#} v:=\left.\frac{d}{d t}\right|_{t=0}(u+t v)$. Then we may identify $\pi^{*} T M$ with $V M$ using \# as follows: for $(u, v) \in \pi^{*} T M, u$ and $v$ are in the same tangent space to $M$, so $u_{\sharp} v$ makes sense, and $(u, v) \leftrightarrow u_{\#} v$ is the required bundle identification.

Suppose $\xi \rightarrow M$ is a vector bundle over $M$; let $\Lambda^{j} \xi$ be the associated bundle over $M$ for which the fiber at $p$ in $M$ is the vector space of alternating $j$-linear forms on the fiber $\xi_{p}$ in $\xi$ over $p$.

Given $\omega \in \Lambda^{j} \xi$ and $\mu \in \Lambda^{\ell} \xi$, both over the same point $p$ in $M$, we have as usual the $(j+\ell)$-form

$$
\omega \wedge \mu:=\frac{1}{j ! \ell !} \sum_{\sigma} \operatorname{sgn}(\sigma)(\omega \otimes \mu) \circ \sigma
$$

where the sum is taken over the symmetric group on $j+\ell$ letters.

Recall that $\mathfrak{v} M=\Gamma T M$, and let $A^{r}(\xi)$ be the $F M$-module of alternating $r$ linear forms on $\mathfrak{v} M$ with values in $\Gamma \xi$. If $\nabla: \mathfrak{v} M \times \Gamma \xi \rightarrow \Gamma \xi$ is a covariant derivative operator in $\xi$, then we have the standard exterior derivative operator $d^{r}: A^{r}(\xi) \rightarrow A^{r+1}(\xi)$ with respect to $\nabla$ defined by

$$
\left[d^{\nabla} \omega\right](X):=\nabla_{X} \omega
$$

for $\omega \in A^{0}(\xi)$, and by

$$
\begin{aligned}
{\left[d^{\nabla} \omega\right]\left(X_{0}, \cdots, X_{r}\right):=} & \sum_{i}(-1)^{i} \nabla_{X_{i}}\left(\omega\left(X_{0}, \cdots, \hat{X}_{i}, \cdots, X_{r}\right)\right) \\
& +\sum_{i<j}(-1)^{i+j} \omega\left(\left[X_{i}, X_{j}\right], X_{0}, \cdots, \hat{X}_{i}, \cdots, \hat{X}_{j}, \cdots, X_{r}\right)
\end{aligned}
$$

for $\omega \in A^{r}(\xi), r \geq 1$.

Notice that for an arbitrary covariant derivative $\nabla$ in an arbitrary bundle $\xi$, $d^{\nabla} d^{\nabla}$ does not have to vanish; a bundle $\xi$ is said to be flat with respect to $\nabla$ if and only if $d^{\nabla} d^{\nabla}=0$. Example: $M \times R$ is flat with respect to its canonical connection.

We will be using modules of the form $A^{r}\left(\Lambda^{j} \xi\right)$, and we need a sort of double wedge product. Given $\phi \in A^{r}\left(\Lambda^{j} \xi\right), \psi \in A^{s}\left(\Lambda^{l} \xi\right)$, and $X_{1}, \cdots, X_{r+s} \in \mathfrak{v} M$, we define the form $\phi \wedge \psi\left(X_{1}, \cdots, X_{r+s}\right) \in \Gamma \Lambda^{j+\ell} \xi$ to be $(r ! s !)^{-1} \sum_{\sigma} \operatorname{sgn}(\sigma) \phi\left(X_{\sigma_{1}}\right.$, 
$\left.\cdots, X_{\sigma_{r}}\right) \wedge \psi\left(X_{\sigma_{r+1}}, \cdots, X_{\sigma_{r+s}}\right)$, where as usual the sum is taken over the symmetric group on $r+s$ letters.

$d^{\nabla}$ and $\wedge$ have the following usual properties:

(1) $d^{\nabla}$ is natural: $f^{*}\left(d^{\nabla} \omega\right)=d^{f^{*}}\left(f^{*} \omega\right)$.

(2) $\wedge$ is associative.

(3) For $\phi \in A^{r}\left(\Lambda^{j} \xi\right)$ and $\psi \in A^{s}\left(\Lambda^{\ell} \xi\right), \phi \wedge \psi=(-1)^{r s+j \ell} \psi \wedge \phi$.

(4) For $\phi$ and $\psi$ as in (3), $d^{\nabla}(\phi \wedge \psi)=\left(d^{\nabla} \phi\right) \wedge \psi+(-1)^{r} \phi \wedge d^{\nabla} \psi$.

We will use the canonical identification of functions on $M^{n}$ with $n$-forms to convert elements of $A^{r}\left(\Lambda^{n} T M\right)$ into ordinary differential $r$-forms on $M$; this will allow us to integrate such $(r, n)$-forms without further comment. More generally, any $(r, r)$-form restricted to an $r$-plane can be thought of as a number; an example of this occurs in the next paragraph.

A curvature form on a Euclidean vector space $\mathscr{V}$ is a 2 -form $\Omega$ on $\mathscr{V}$ with values in 2-forms on $\mathscr{V}$ such that $\Omega$ satisfies the Jacobi identity in the first three arguments. Assume that $\mathscr{V}$ is even-dimensional, $\operatorname{dim} \mathscr{V}=2 j$. The algebraic Hopf conjecture is that if $-\Omega$ is nonnegative (resp. positive) on all 2planes in $\mathscr{V}$, then $(-\Omega)^{j}$ is nonnegative (resp. positive) on $\mathscr{V}$.

Notice that $-\Omega$ restricted to 2 -planes in $\mathscr{V}$ is just the usual sectional curvature function. The conclusion of the Hopf conjecture is true under certain extra algebraic conditions which have no geometric significance in our situation.

Define the curvature form $\Omega \in A^{2}\left(\Lambda^{2} T M\right)$ on $M$ with respect to $\nabla$ by

$$
\Omega(X, Y)(U, V):=\left\langle d^{\nabla} d^{\nabla} U(X, Y), V\right\rangle=\langle R(X, Y) U, V\rangle
$$

for vector fields $X, Y, U$ and $V$ on $M$, where $R$ is the usual curvature tensor on $M$. The Bianchi identity now takes the form

$$
d^{\nabla} \Omega=0
$$

Pull $\langle$,$\rangle and \nabla$ back to $\pi^{*} T M=V M$, and let $\Lambda=\Lambda(V M)=\Lambda\left(\pi^{*} T M\right)$.

Let $I$ be the "position vector field" on $T M$ along $\pi$ :

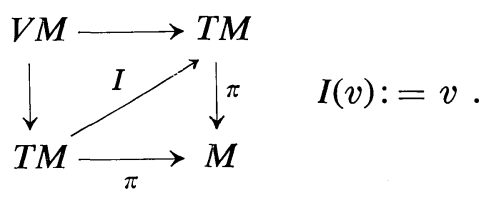

Let $\eta \in A^{0}\left(\Lambda^{1}\right)$ be the canonical 1-form defined by

$$
\eta(s):=\left\langle\pi^{*} I, s\right\rangle
$$

for $s$ in $V M$. Suppose that $s=u_{\sharp} v$; then

$$
\eta(s)=\left.\left\langle\pi^{*} I, s\right\rangle\right|_{u}=\left\langle\left(\pi^{*} I\right) u, u_{\sharp} v\right\rangle=\left\langle u_{\sharp} u, u_{\sharp} v\right\rangle=\langle u, v\rangle
$$


by the definition of $\langle$,$\rangle on T M$ as the pullback of $\langle$,$\rangle on M$.

Next we define the connection 1-form on $V M$ by

$$
\theta:=d^{\nabla} \eta \in A^{1}\left(\Lambda^{1}\right)
$$

For $v \in T T M$ and $s \in V M, \theta(v)(s)=\left(\nabla_{v} \eta\right)(s)=\left\langle\nabla_{v} \pi^{*} I, s\right\rangle$. We now mention some facts which will be needed below.

(1) If $v$ is vertical on $T M$, then $\nabla_{v} I=v$, and therefore $\theta(s)(t)=\langle s, t\rangle$ for $s$ and $t$ in $V M$.

(2) If $v$ is tangent to $T_{1} M$, then $\theta(v)\left(\pi^{*} I\right)=0$.

(3) Consider $d^{\nabla} \theta \in A^{2}\left(\Lambda^{1}\right)$; for $u$ and $v \in T T M$ and $s \in V M$,

$$
d^{v} \theta(u, v)(s)=\pi^{*} \Omega(u, v)\left(\pi^{*} I, s\right) .
$$

(4) $\pi^{*} \Omega(u, v)=0$ for vertical vectors $u, v \in T T M$.

For $1 \leq i \leq k$, let

$$
\begin{aligned}
\Pi_{i} & :=\eta \wedge \theta^{2 i-1} \wedge \pi^{*} \Omega^{k-i} \in A^{n-1}\left(\Lambda^{n}\right), \\
c_{i} & :=\frac{(-1)^{k+i+1}(i-1) !}{\pi^{k} 2^{k-i+1}(k-i) !(2 i-1) !} .
\end{aligned}
$$

Define

$$
\Pi:=\sum_{i=1}^{k} c_{i} \Pi_{i}
$$

The key points in the classical Chern argument [2] are the following: first of all, on $T_{1} M$,

$$
d^{\nabla} \Pi=\frac{(-1)^{k}}{k !(2 \pi)^{k}} \pi^{*} \Omega^{k}
$$

second, on the unit sphere $S_{p}^{n-1}$ in $M_{p}, \Pi$ is a constant times the volume form, and in particular

$$
\int_{S_{p}^{n-1}} \Pi=-1
$$

These equalities are proven by choosing special bases and then applying the definitions and facts (1)-(4).

Define the Euler form on $M$ by

$$
\chi:=\frac{(-1)^{k}}{k !(2 \pi)^{k}} \Omega^{k} \in A^{n}\left(\Lambda^{n}\right) .
$$

$\pi^{*} \chi$ is exact on $T_{1} M . \chi$ is often called the Chern integrand. 
Let $H$ be an $(n-1)$-dimensional submanifold of $M$ equal to a level set $f^{-1}(c)$ for some $f$ in $F M$ and some regular value $c$ of $f$. The Hessian form of $f$ at $p$ is the symmetric bilinear form $h_{p}: M_{p} \times M_{p} \rightarrow R$ defined by $h_{p}(u, v)$ $:=\left\langle\nabla_{u} \nabla f, v\right\rangle$ for $u$ and $v$ in $M_{p}$. Notice that for $p$ in $H, h_{p}$ restricted to $H_{p} \times H_{p}$ is just $\|\nabla f(p)\|$ times the second fundamental form of $H$ at $p$ with respect to the unit normal field $\nabla f /\|\nabla f\|$ on $H$; call this restricted unit normal field $X$. We want to estimate $\int_{H} X^{*} \Pi$. To do this we first evaluate $g(p)$, where $g$ is the function on $H$ determined by the identification

$$
\left[X^{*} \Pi\right]_{p} \simeq g(p) \cdot d_{p}\left(\operatorname{vol}_{H}\right)
$$

where $d\left(\operatorname{vol}_{H}\right)$ is the volume form on $H$. Let $u_{0}:=X_{p}$, and let $u_{1}, \cdots, u_{n-1}$ be the principal curvature directions of $H$ at $p$ with principal curvatures $\lambda_{1}$, $\cdots, \lambda_{n-1}$ respectively. Then

$$
\begin{aligned}
& g(p)= g(p) \cdot d_{p}\left(\operatorname{vol}_{H}\right)\left(u_{1}, \cdots, u_{n-1}\right) \\
&= X^{*} \Pi\left(u_{1}, \cdots, u_{n-1}\right)\left(u_{0}, \cdots, u_{n-1}\right) \\
&= \sum_{i=1}^{k} c_{i} X^{*}\left(\eta \wedge \theta^{2 i-1} \wedge \Omega^{k-i}\right)\left(u_{1}, \cdots, u_{n-1}\right)\left(u_{0}, \cdots, u_{n-1}\right) \\
&=\sum c_{i} \sum \operatorname{sgn}(\sigma) \operatorname{sgn}(\tau) \frac{1}{[(n-2 i) !]^{2}}\left\langle X, u_{\tau_{0}}\right\rangle\left\langle\nabla_{\left.X_{*} u_{\sigma_{1}} X_{\#} X, X_{\#} u_{\tau_{1}}\right\rangle}\right. \\
& \cdots X^{*} \pi^{*} \Omega^{k-i}\left(u_{\sigma_{2 i}}, \cdots, u_{\sigma_{n-1}}\right)\left(u_{\tau_{2 i}}, \cdots, u_{\tau_{n-1}}\right),
\end{aligned}
$$

where the inner sum is taken over all $\sigma \in S_{n-1}$ acting on $\{1, \cdots, n-1\}$, and $\tau \in S_{n}$ acting on $\{0, \cdots, n-1\}$, and everything is evaluated at $p$. Since $\left\langle X, u_{\tau_{0}}\right\rangle=\delta_{0, \tau_{0}}$ by the definition of the $u_{i}$, and

$$
\left\langle\nabla_{X_{*} u_{1}} X \text { \# } X, X_{\sharp} u_{\tau_{1}}\right\rangle=\left\langle\nabla_{u_{\sigma_{1}}} X, u_{\tau_{1}}\right\rangle=\delta_{\sigma_{1}, \tau_{1}} \lambda_{\sigma_{1}},
$$

we have

$$
g(p)=\sum_{1}^{k}(2 i-1) ! c_{i} \sum \lambda_{j_{1}} \cdots \lambda_{j_{2 i-1}} \Omega^{k-i}
$$

where $\Omega^{k-i}$ is evaluated on $u_{\ell_{1}}, \cdots, u_{\ell_{n-2} i}$, and the inner sum is taken over all $j_{1}<\cdots<j_{2 i-1}$ and $\ell_{1}<\cdots<\ell_{n-2 i}$ such that $\left\{j_{1}, \cdots, j_{2 i-1}, \ell_{1}, \cdots, \ell_{n-2 i}\right\}=$ $\{1, \cdots, n-1\}$.

If the boundaries of the sets $C_{t}$ were $C^{2}$ for all sufficiently large $t$, the remaining work would be simple; unfortunately, the boundary of a totally convex set is in general only continuous, and the set of points of non-differentiability can in fact be dense in the boundary. The nicest way around the difficulty would be to approximate each set by a totally convex set with $C^{2}$ boundary; so far we have been unable to prove that this is possible in general. For 
our purposes it is sufficient to have an approximating set with a differentiable boundary which is only close to being totally convex.

Recall that on a manifold a convex function is a continuous function $f: M \rightarrow R$ such that for every geodesic $c$ in $M, f \circ c$ is a convex function on $R$ in the standard sense, that is, given real numbers $a$ and $b$,

$$
f \circ c(s a+(1-s) b) \leq s f \circ c(a)+(1-s) f \circ c(b)
$$

for $0 \leq s \leq 1$. All the sublevel sets of a convex function are totally convex.

In our situation, the function $f: M \rightarrow R$ such that

$$
f(p)= \begin{cases}0 & \text { if } p \in C_{0}, \\ t & \text { if } p \in \partial C_{t} \text { for } t>0\end{cases}
$$

is convex. This follows since on $C_{t},\left[p \mapsto-\rho\left(p, \partial C_{t}\right)\right]$ is a convex function (see [1]), and $f(p)=t-\rho\left(p, \partial C_{t}\right)$ for $p$ in $C_{t}$.

In attempting to find a $C^{\infty}$ convex approximation for $f$ one is naturally led to the idea of convolving $f$ with a $C^{\infty}$ bump function. The result fails to be convex, but is close in the sense of the following lemma.

Lemma 7. Given $K$ compact in $M$, choose $\varepsilon>0$ as in Lemma 3. Suppose $f: M \rightarrow R$ is convex. Then there exist an open neighborhood $U$ of $K$ and $a$ family $\left\{f_{r} \in F U \mid r \in(0, \varepsilon)\right\}$ such that

(1) the functions $\left.f_{r}\right|_{K}$ converge uniformly to $\left.f\right|_{K}$ as $r \rightarrow 0$,

(2) $f_{r}$ approaches $f$ monotonically from above,

(3) $\varliminf_{r \rightarrow 0}\left(\inf _{u \in T_{1} K} h_{f_{r}}(u, u)\right) \geq 0$ where $h_{f_{r}}$ is the Hessian form of $f_{r}$.

A complete proof of this result has been given by Greene and $\mathrm{Wu}$ in [5], so we will give no more than the explicit construction of the functions $f_{r}$ for later use.

Let $\mu: R \rightarrow R$ be a nonnegative $C^{\infty}$ bump function with support in $[-1,1]$ which is constant near zero and such that

$$
\int_{R^{n}} \mu(\|v\|) d v=1
$$

Define

$$
f_{r}(p):=\frac{1}{r^{n}} \int_{M_{p}} f \circ \exp (v) \mu\left(\frac{\|v\|}{r}\right) d v
$$

Theorem. Let $M$ be a complete oriented Riemannian manifold of nonnegative sectional curvature and even dimension $2 k$. Assume that the algebraic Hopf conjecture holds on $M$. Then the total curvature of $M$ exists and is bounded between zero and the Euler characteristic of $M$.

Proof. For each positive integer $i$, let $\varepsilon_{i}>0$ be a constant as chosen in 
Lemma 3 for the set $C_{i}$ in the filtration of $M$ by compact totally convex sets $C_{t}$. Fix a positive integer $i \geq 2$; let $\left\{f_{r} \mid r \in\left(0, \varepsilon_{i}\right)\right\}$ be a sequence of $C^{\infty}$ functions on a neighborhood $U$ of $C_{i}$ as given by Lemma 7 for the function $f$ indexing the sets $C_{t}$.

First we prove that for $r$ sufficiently small, $f_{r}$ has no critical points off of a neighborhood of $C_{0}$ in $C_{1}$, and $f_{r}^{-1}(-\infty, i]$ is homeomorphic to $C_{i}$.

Choose a positive $\delta<\min \left\{\varepsilon_{i}, 1\right\}$ for the set $C_{i}$ as in Lemma 4. Let $i-\delta<a^{\prime}<a<i$. Then we have a continuous geodesic contraction of $C_{i}$ onto $C_{a^{\prime}}$ and the distance function $d$ from $C_{i}$ to $C_{a^{\prime}}$ is $C^{1}$ on $C_{i}-C_{a^{\prime}}$. For each $p$ in $\partial C_{a}$, there is a unique geodesic $\gamma_{p}$ through $p$ such that $\gamma_{p}(0)=p$ and $\dot{\gamma}_{p}(0)=\nabla d(p) ; \gamma_{p}$ is a reparametrization of one of the geodesics given by the contraction of $C_{i}$ onto $C_{a^{\prime}} . \partial C_{a^{\prime}}$ is transverse to $\gamma_{p}$, and therefore $f$ is strictly increasing along $\gamma_{p}$. By continuity, for each $p$ there is a cone $W_{p}$ of normal geodesics through $p$ about $\gamma_{p}$ such that $f$ is strictly increasing along each $c$ in $W_{p}$. We may assume that there is some positive number $\alpha$ such that for all $p$, $W_{p}$ is the set of all normal geodesics on the interval $[-\beta, 1]$ which have $c(0)=p$ and $\Varangle\left(\dot{c}(0), \dot{\gamma}_{p}(0)\right) \leq \alpha$, where $\beta$ is some positive number less than $a-a^{\prime}$. Let $W:=\bigcup_{p \in \partial C_{a}} W_{p}$. Then $\left\{\left.f \circ c\right|_{[-\beta, 1]} \mid c \in W\right\}$ is a continuous compact family of strictly increasing functions, all of which are convex and have the value $a$ at 0 . All of the difference quotients

$$
\frac{1}{t}[f \circ c(t)-a], \quad t \neq 0, c \in W,
$$

are positive, and it is a straightforward proof that the difference quotients for $t>0$ have a uniform positive lower bound $\kappa$. Then by monotonicity of the difference quotients of a convex function,

$$
\frac{f \circ c(t)-f \circ c(s)}{t-s} \geq \kappa
$$

if $0 \leq s<t$ and $c \in W$.

Furthermore, there exists a positive number $r_{0}$ such that for all $c$ in $W$, the difference quotients of $f$ along every curve of the form $\left[t \mapsto \exp _{c(t)} V(t)\right]$ are greater than or equal to $\frac{1}{2} \kappa$, where each $V$ is a parallel vector field along $c$ for which $\|V(t)\|<r_{0}$. We may assume that $r_{0}<\varepsilon_{i}$.

Now let $c=\gamma_{p}$ for some $p$ in $\partial C_{a}$. We want to calculate $\left(f_{r} \circ c\right)^{\prime}(t)$ for $t>0$. Now $r^{n}\left(f_{r} \circ c(s)-f_{r} \circ c(t)\right)$ is equal to

$$
\begin{array}{r}
\int_{M_{c(s)}} f \circ \exp _{c(s)}(v) \mu\left(\frac{\|v\|}{r}\right) d v-\int_{M_{c}(t)} f \circ \exp _{c(t)}(v) \mu\left(\frac{\|v\|}{r}\right) d v \\
=\int_{M_{c}(t)}\left(f \circ \exp _{c(s)} V(s)-f \circ \exp _{c(t)} V(t)\right) \mu\left(\frac{\|v\|}{r}\right) d v
\end{array}
$$


where $V$ is the parallel vector field along $c$ with $V(t)=v$. Thus, if $r<r_{0}$, then

$$
f_{r} \circ c(s)-f_{r} \circ c(t) \geq \frac{1}{2}(s-t) \kappa,
$$

and therefore $\left(f_{r} \circ c\right)^{\prime}(t) \geq \frac{1}{2} \kappa$. But this means that $\left\langle\nabla f_{r}, \nabla d\right\rangle$ is positive for $r<r_{0}$; notice that by the same argument we can prove that off of a small neighborhood of $C_{0}$, the derivative of $f_{r}$ in a direction transverse to the boundary of each set $C_{t}$ is positive for sufficiently small $r$. In particular, for small $r$ we may use the gradient flow of some distance function $d$ to obtain a homeomorphism between $C_{i}$ and $\Xi_{r}:=f_{r}{ }^{-1}(-\infty, i]$. This is a crucial step in the proof of the theorem since it allows us to equate the Euler characteristic of $\Xi$ with the Euler characteristic of $M$ :

$$
\chi_{\varepsilon_{r}}=\chi_{C_{i}}=\chi_{S}=\chi_{M} .
$$

We will also use the fact that $f_{r}$ has no critical points near $\partial C_{i}$ since that means that $\Xi_{r}=f_{r}{ }^{-1}(i)$ is a submanifold.

From here on the proof is almost identical to what it would have been if the boundary of $C_{i}$ had been $C^{2}$.

We may approximate $\nabla f_{r}$ by a $C^{\infty}$ vector field $V_{r}$ which equals $\nabla f_{r}$ near $\partial \Xi_{r}$ and has only isolated zeros. By the Poincaré-Hopf Theorem [9], the sum of the indices of the zeros of $V_{r}$ is just the Euler characteristic of $\Xi_{r}$, that is, $\chi_{M}$. Let $Y_{r}:=V_{r} /\left\|V_{r}\right\|$ on $\Xi_{r}-$ zeros of $\left.V_{r}\right\}$.

Then

$$
\int_{\Xi_{r}} \chi=\int_{\partial \Xi_{r}} Y_{r}^{*} \Pi+\chi_{M}
$$

But

$$
C_{i}=\overline{\bigcup_{r>0} \Xi_{r}}
$$

since $f_{r} \geq f$, and then

$$
\int_{C_{i}} \chi=\lim _{r \rightarrow 0} \int_{\Xi_{r}} \chi
$$

In particular, the limit of $\int_{\partial \varepsilon_{r}} Y_{r}^{*} \Pi$ exists as $r \rightarrow 0$. As before, $\int_{\partial \varepsilon_{r}} Y_{r}^{*} \Pi=$ $\int_{\partial \varepsilon_{r}} g_{r}(p) \cdot d_{p}\left(\operatorname{vol}_{\partial \varepsilon_{r}}\right)$, and

$$
g_{r}(p)=-\sum_{m=1}^{k} \frac{(m-1) !}{2^{k-m+1} \pi^{k}(k-i) !} \sum \lambda_{j_{1}} \cdots \lambda_{j_{2 m-1}}(-\Omega)^{k-i}
$$

where $(-\Omega)^{k-i}$ is evaluated on the plane spanned by $v_{\ell_{1}}$ through $v_{\ell_{n-2 m}}$, and 
the inner sum is taken over all $j_{1}<\ldots<j_{2 m-1}$ and $\ell_{1}<\ldots<\ell_{n-2 m}$ such that $\left\{j_{1}, \cdots, j_{2 m-1}, \ell_{1}, \cdots, \ell_{n-2 m}\right\}=\{1, \cdots, n-1\}$. Here $v_{1}, \cdots, v_{n-1}$ are the principal curvature directions of $\partial \Xi_{r}$ at $p$ with corresponding principal curvatures $\lambda_{1}, \cdots, \lambda_{n-1}$. By the choice of the functions $f_{r}$ and the fact that the Hessian form of $f_{r}$ at $p$ is just $\left\|\nabla f_{r}(p)\right\|$ times the second fundamental form of $\partial \Xi_{r}$ at $p$, we see that $\varliminf_{r \rightarrow 0} \lambda_{j_{1}} \cdots \lambda_{j_{2 m-1}} \geq 0$. Since we have assumed that the Hopf conjecture is true on $M,(-\Omega)^{k-i}$ is nonnegative. The result is that $\varlimsup_{r \rightarrow 0} g_{r}(p) \leq 0$, and consequently,

$$
\lim _{r \rightarrow 0} \int_{\partial E_{r}} Y_{r}^{*} \Pi \leq 0
$$

Here we use the fact that $\left\{\operatorname{vol}\left(\partial \Xi_{r}\right)\right\}_{r>0}$ is bounded, which is easily proven using the basic Hausdorff measure theory. But then,

$$
\int_{C_{i}} \chi=\lim _{r \rightarrow 0} \int_{\Xi_{r}} \chi=\lim _{r \rightarrow 0} \int_{\partial \varepsilon_{r}} Y_{r}^{*} \Pi+\chi_{M} \leq \chi_{M} .
$$

Again by the Hopf conjecture, $0 \leq \int_{C_{i}} \chi \leq \int_{C_{i+1}} \chi$ for all $i$. Therefore $\int_{M} \chi$ exists and $0 \leq \int_{M} \chi \leq \chi_{M}$.

\section{Some remarks}

Cohn-Vossen classified the complete noncompact surfaces of nonnegative curvature [4]; for such a surface $M$, the soul $S$ is either a point or is diffeomorphic to $S^{1}$. If $S$ is a point, then $M$ is diffeomorphic to $R^{2}$, and Cohn-Vossen proved that the total curvature is bounded between 0 and 1 ; these estimates are sharp. If $S$ is diffeomorphic to $S^{1}$, then $M$ is isometric to one of the two flat line boundles over $S$ and has zero total curvature.

Our result gives a new proof of the Cohn-Vossen total curvature result.

Cheeger and Gromoll classified the complete noncompact 3-manifolds of nonnegative curvature using the soul theorem [1]; the soul is diffeomorphic to one of the following: a point, $S^{1}, S^{2}, R P^{2}$, the flat torus, or the flat Klein bottle. In addition, they proved that if the soul $S$ of an arbitrary complete Riemannian manifold of nonnegative curvature has codimension 1 , then $M$ is isometrically a flat line bundle over $S$.

Gromoll and Meyer proved that if the curvature of $M$ is everywhere positive, then the soul is a point (see [7]). From this they proved, for $n \neq 3$ or 4 , that $M$ and $R^{n}$ are diffeomorphic; our result implies directly that for any manifold for which the soul is a point, the manifold is diffeomorphic to $R^{n}$ and the total curvature, if it exists, is bounded above by 1 .

We will assume that all our manifolds are oriented; by considering the orientation covering, similar information can be gotten for nonoriented mani- 
folds. No attempt will be made to classify anything, but we will make some comments about the cases of dimensions 4 and 6 .

Dimension 4. The only possible souls with positive Euler characteristic are a point and $S^{2}$. Thus we see immediately that $0 \leq \int_{M} \chi \leq 2$.

If the soul is diffeomorphic to $S^{1}$, then the total curvature of $M$ is zero.

Suppose $\operatorname{dim} S=2$. If $S$ is the flat torus, then $M$ is locally isometrically trivial over $S$ (see [1]), and in such a case the Chern integrand is identically zero. If $S$ is diffeomorphic to $S^{2}$ and the total curvature of $M$ is positive, then $M$ is not isometrically the product $S^{2} \times R^{2} ; M$ may still be the product of $S^{2}$ and some nonnegatively curved surface diffeomorphic to $R^{2}$, and there are examples where $M$ is not even locally isometrically a product, e.g., $T S^{2}$.

If $\operatorname{dim} S=3$, then $\chi_{M}$ is of course zero, as is the case whenever the soul of $M$ is odd-dimensional. In addition, in this case $M$ is isometrically a flat line bundle over $S$ with zero Chern integrand. Despite these restrictions there are still many possibilities for $M$, since there are already 6 flat compact orientable 3-manifolds [11].

Dimension 6. Here we cannot in general say that the total curvature exists, but since the boundary terms involve only the first and second powers of the curvature form, we do know that

$$
\varlimsup_{i \rightarrow \infty} \int_{C_{i}} \chi \leq \chi_{M}
$$

Even if the total curvature does exist, without more information we have no guarantee that it will be nonnegative. The number of manifolds here will be quite large since in the flat case alone there are very many manifolds of dimension less than six. Notice however that if $S$ is flat, then the Chern integrand is identically zero on $M$ since locally $M$ has a flat factor [1].

\section{References}

[1] J. Cheeger \& D. Gromoll, On the structure of complete manifolds of nonnegative curvature, Ann. of Math. 96 (1972) 413-443.

[2] S. S. Chern, A simple intrinsic proof of the Gauss-Bonnet formula for closed Riemannian manifolds, Ann. of Math. 45 (1944) 747-752.

[3] - On curvature and characteristic classes of a Riemannian manifold, Abh. Math. Sem. Univ. Hamburg 20 (1956) 117-126.

[4] S. Cohn-Vossen, Kürzeste Wege und Totalkrümmung auf Flächen, Composito Math. 2 (1935) 69-133.

[ 5 ] R. Greene \& H. Wu, On the subharmonicity and plurisubharmonicity of geodesically convex functions, Indiana Univ. Math. J. 22 (1973) 641-654.

[6] D. Gromoll, W. Klingenberg \& W. Meyer, Riemannsche Geometrie im Grossen, Springer, Berlin, 1968.

[ 7 ] D. Gromoll \& W. Meyer, On complete open manifolds of positive curvature, Ann. Math. 90 (1969) 75-90.

[ 8 ] J. Milnor, Morse theory, Annals of Math. Studies, No. 51, Princeton University Press, Princeton, 1963. 
[9] — Topology from the differentiable viewpoint, University Press of Virginia, Charlottesville, 1965.

[10] J. Munkres, Elementary differential topology, Annals of Math. Studies, No. 54, Princeton University Press, Princeton, 1963.

[11] J. Wolf, Spaces of constant curvature, McGraw-Hill, New York, 1966.

State University of New York, Stony Brook 